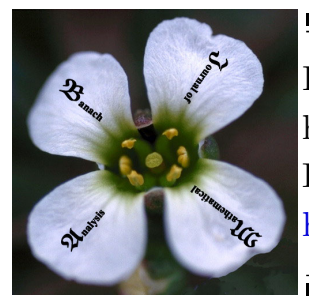

Banach J. Math. Anal. 9 (2015), no. 1, 159-165

http://doi.org/10.15352/bjma/09-1-13

ISSN: $1735-8787$ (electronic)

http://projecteuclid.org/bjma

\title{
HARMONIC FUNCTIONALS ON CERTAIN BANACH ALGEBRAS
}

\author{
MEHDI NEMATI
}

Communicated by N. Gronbæk

\begin{abstract}
In this paper, we study the concept of harmonic functionals for certain Banach algebras such as generalized Fourier algebras. For a nonzero character $\phi$ on Banach algebra $\mathcal{A}$, we also characterize the concept of $\phi$-amenability in terms of harmonic functionals. Finally, for a locally compact group $G$ we investigate the space $H_{\sigma, x}$ of $\sigma$-harmonic functionals in the dual of generalized Fourier algebra $A_{p}(G)$. The main result states that $G$ is first countable if and only if $\sigma$ is adapted if and only if $H_{\sigma, x}=\mathbb{C} \phi_{x}$.
\end{abstract}

\section{INTRODUCTION AND PRELIMINARIES}

For a locally compact group $G$ and $1<p<\infty$, Herz [6] introduced the generalized Fourier algebra of $G$ denoted by $A_{p}(G)$. Elements of $A_{p}(G)$ can be represented, nonuniquely, as $u=\sum_{i=1}^{\infty}\left(f_{i} * \check{g}_{i}\right)$, where $f_{i} \in L^{p}(G), g_{i} \in L^{q}(G), \frac{1}{p}+\frac{1}{q}=1$, $\check{g}(x)=g\left(x^{-1}\right)$ and $\sum_{i=1}^{\infty}\left\|f_{i}\right\|_{p}\|\| g_{i} \|_{q}<\infty$. Then

$$
\|u\|_{A_{p}}=\inf \left\{\sum_{i=1}^{\infty}\left\|f_{i}\right\|_{p}\|\| g_{i} \|_{q}: u=\sum_{i=1}^{\infty}\left(f_{i} * \check{g}_{i}\right)\right\}
$$

determines a norm on $A_{p}(G)$. When $p=2, A_{p}(G)$ coincides with the Fourier algebra $A_{2}(G)$ introduced by Eymard [4].

For $1<p<\infty$ we denote by $\mathcal{L}\left(L^{p}(G)\right)$ the space of all continuous linear operators on $L^{p}(G)$, equipped with the usual operator norm $\|\cdot\|_{o p}$, and let

Date: Received: Feb. 12, 2014; Accepted: Apr. 21, 2014.

2010 Mathematics Subject Classification. Primary 43A07, 43A15; Secondary 46J10.

Key words and phrases. Generalized Fourier algebras, harmonic functionals, $\phi$-mean. 
$\lambda_{p}: M(G) \rightarrow \mathcal{L}\left(L^{p}(G)\right)$ be the left regular representation of the measure algebra $M(G)$ on $L^{p}(G)$ defined by $\lambda_{p}(\mu)(f)=\mu * f$, where $\mu \in M(G), f \in$ $L^{p}(G)$ and $\mu * f=\int_{G} f\left(y^{-1} x\right) d \mu(y)$. Let $P M_{p}(G)$ be the weak*-closure of $\lambda_{p}(M(G))$ in $\mathcal{L}\left(L^{p}(G)\right)$, where the closure is with respect to the weak* topology $\sigma\left(\mathcal{L}\left(L^{p}(G)\right), L^{p}(G) \widehat{\otimes} L^{q}(G)\right)$. The space $P M_{p}(G)$ called the space of $p$-pseudomeasures on $G$. It is well known that $P M_{p}(G)$ can be identified with the dual of the generalized Fourier algebra $A_{p}(G)$. When $\mu \in M(G)$ the dual action of $\lambda_{p}(\mu)$ on $A_{p}(G)$ is defined by $\lambda_{p}(\mu)(u)=\int_{G} u(x) d \mu(x)$ for all $u \in A_{p}(G)$. With the usual operations of pointwise addition and multiplication, $A_{p}(G)$ is a commutative semisimple regular and Tauberian Banach algebra.

Let $M A_{p}(G)$ be the multiplier algebra of $A_{p}(G)$; that is, the set of all continuous functions $v$ on $G$ such that $v u \in A_{p}(G)$ for all $u \in A_{p}(G)$. With the multiplier norm

$$
\|v\|_{M}=\inf \left\{\|u v\|_{A_{p}}: u \in A_{p}(G),\|u\|_{A_{p}} \leq 1\right\}
$$

$M A_{p}(G)$ is a Banach algebra containing $A_{p}(G)$ as an ideal with decreasing norms $\|\cdot\|_{M} \leq\|\cdot\|_{A_{p}}$. There is a natural $M A_{p}(G)$-module action on $P M_{p}(G)$ defined by $\langle v \cdot T, u\rangle=\langle T, u v\rangle$ for all $u \in A_{p}(G), v \in M A_{p}(G)$ and $T \in P M_{p}(G)$.

Let $\mathcal{A}$ be a Banach algebra. We denote by $\Delta(\mathcal{A})$ the set of all non-zero characters, bounded multiplicative linear functionals on $\mathcal{A}$. For $\phi \in \Delta(\mathcal{A})$, Kaniuth, Lau and Pym [11, 12] introduced and investigated a notion of amenability for Banach algebras called $\phi$-amenability; see also $[1,2,9,19]$. In fact, $\mathcal{A}$ is said to be $\phi$-amenable if there exists $m \in \mathcal{A}^{* *}$ such that $m(\phi)=1$ and $m(f \cdot a)=\phi(a) m(f)$ for all $f \in \mathcal{A}^{*}$ and $a \in \mathcal{A}$, where $f \cdot a \in \mathcal{A}^{*}$ is defined by $(f \cdot a)(b)=f(a b)$ for all $b \in \mathcal{A}$. Any such $m$ is called a $\phi$-mean. An element $a$ of $\mathcal{A}$ is called $\phi$-maximal if it satisfies $\|a\|=\phi(a)=1$. Let $S_{\phi}^{\mathcal{A}}$ denote the collection of all $\phi$-maximal elements of $\mathcal{A}$. It is easy to see that $S_{\phi}^{\mathcal{A}}$ is a convex semigroup. We denote by $\overline{S_{\phi}^{\mathcal{A}}} w^{*}$ the weak*-closure of $S_{\phi}^{\mathcal{A}}$ in $\mathcal{A}^{* *}$.

Let $\left(\mathcal{B},\|\cdot\|_{\mathcal{B}}\right)$ be a Banach algebra containing the Banach algebra $\left(\mathcal{A},\|\cdot\|_{\mathcal{A}}\right)$ as a two-sided ideal with decreasing norms $\|\cdot\|_{\mathcal{B}} \leq\|\cdot\|_{\mathcal{A}}$ and let $\phi \in \Delta(\mathcal{A})$. Then we can extend $\phi$ to an element in $\Delta(\mathcal{B})$ whic is equal to $\phi$ on $\mathcal{A}$, we denote this extension still by $\phi$. It is easy to see that $S_{\phi}^{\mathcal{A}} \subseteq S_{\phi}^{\mathcal{B}}$. For each $b \in S_{\phi}^{\mathcal{B}}$, we denote by $I_{b, \phi}$ the norm closure of the set $\{a-a b: a \in \mathcal{A}\}$ in $\mathcal{A}$ and set $I_{\phi}=\{a \in \mathcal{A}: \phi(a)=0\}$. Following [3], the elements of $H_{b, \phi}:=I_{b, \phi}{ }^{\perp}$ are called $b$-harmonic functionals. We note that

$$
H_{b, \phi}=\left\{f \in \mathcal{A}^{*}: b \cdot f=f\right\} .
$$

It is well known that $\Delta\left(A_{p}(G)\right)$ can be canonically identified with $G$. More precisely, the map $x \rightarrow \phi_{x}$, where $\phi_{x}(u)=u(x)$ for $u \in A_{p}(G)$, is a homeomorphism from $G$ onto $\Delta\left(A_{p}(G)\right)$. For each $x \in G$ we set

$$
S_{x}^{A}=\left\{u \in A_{p}(G):\|u\|_{A_{p}}=u(x)=1\right\}
$$

and

$$
S_{x}^{M}=\left\{v \in M A_{p}(G):\|v\|_{M}=v(x)=1\right\}
$$


Let $e \in G$ be the identity element of $G$. We recall from [17, Lemma 1.1] that

$$
\overline{S_{e}^{A}} w^{*}=\left\{F \in A_{p}(G)^{* *}:\|F\|=F\left(\phi_{e}\right)=1\right\} .
$$

Now suppose that $x \in G$ and $L_{x}$ is the left translation by $x$ on $A_{p}(G)$; that is, $L_{x} u(y)=u\left(x^{-1} y\right)$ for all $u \in A_{p}(G)$ and $y \in G$. Then as shown in [8, p. 216], $S_{x}^{A}=L_{x}\left(S_{e}^{A}\right)$ and

$$
{\overline{S_{x}^{A}}}^{w^{*}}=\left\{F \in A_{p}(G)^{* *}:\|F\|=F\left(\phi_{x}\right)=1\right\} .
$$

In [18, Lemma 3.1], it is proved that for each $x \in G, A_{p}(G)$ has a $\phi_{x}$-mean in ${\overline{S_{x}^{A}}}^{w^{*}}$. Recall that for each $\sigma \in S_{x}^{M}$, we denote by $I_{\sigma, x}$ the norm closure of the set $\left\{u-u \sigma: u \in A_{p}(G)\right\}$ and set $I_{x}=\left\{u \in A_{p}(G): u(x)=0\right\}$.

In this paper, for a separable Banach algebra $\mathcal{A}$ and $\phi \in \Delta(\mathcal{A})$, among the other things, we show that $\mathcal{A}$ has a $\phi$-mean in ${\overline{S_{\phi}^{\mathcal{A}}}}^{w^{*}}$ if and only if $H_{b, \phi}=\mathbb{C} \phi$ for some $b \in S_{\phi}^{\mathcal{A}}$. Specifically, for a locally compact group $G$, we prove that $G$ is first countable if and only if the space $H_{\sigma, x}$ of $\sigma$-harmonic functionals in $P M_{p}(G)$ is equal to $\mathbb{C} \phi_{x}$ for some $x \in G$ and $\sigma \in S_{x}^{M}$.

\section{HARMONIC FUNCTIONALS}

We commence with the following lemma whose proof is inspired by [10, Theorem 4.1].

Lemma 2.1. Let $\mathcal{A}$ be a separable Banach algebra and let $\phi \in \Delta(\mathcal{A})$. Then the following statements are equivalent.

(a) $\mathcal{A}$ has a $\phi$-mean in $\overline{S_{\phi}^{\mathcal{A}}} w^{*}$.

(b) There is an element $b \in S_{\phi}^{\mathcal{A}}$ such that $\left\|a b^{n}-\phi(a) b^{n}\right\| \rightarrow 0$ for all $a \in \mathcal{A}$.

Proof. $(\mathrm{a}) \Rightarrow(\mathrm{b})$. Suppose that $\left(b_{i}\right)$ is a dense sequence of the unite bale of $\mathcal{A}$ and let $\left(\gamma_{j}\right)$ be a sequence of positive real numbers such that $\sum_{j=1}^{\infty} \gamma_{j}=1$. Choose the increasing sequence $\left(n_{k}\right)$ of positive integers such that $\left(\sum_{j=1}^{k} \gamma_{j}\right)^{n_{k}}<\gamma_{k}$. By assumption and [11, Theorem 1.4] and its proof, there is a net $\left(a_{\alpha}\right) \subseteq S_{\phi}^{\mathcal{A}}$ such that

$$
\left\|a a_{\alpha}-\phi(a) a_{\alpha}\right\| \rightarrow 0
$$

for all for all $a \in \mathcal{A}$. We choose a sequence $\left(a_{m}\right) \subseteq S_{\phi}$, inductively to satisfy

$$
\left\|a_{k_{1}} \ldots a_{k_{\ell}} a_{m}-a_{m}\right\|<\gamma_{m}
$$

for $1 \leq k_{j}<m, 1 \leq j \leq n_{m}$, and

$$
\left\|b_{i} a_{k_{1}} \ldots a_{k_{\ell}} a_{m}-\phi\left(b_{i}\right) a_{m}\right\|<\gamma_{m}
$$

for $1 \leq i, k_{j}<m, 1 \leq j \leq n_{m}$. Then the element

$$
b:=\sum_{m=1}^{\infty} \gamma_{m} a_{m} \in S_{\phi}^{\mathcal{A}}
$$

is the required element. Indeed, the rest of the proof is similar to the proof of [10, Theorem 4.1] and so we omit it. 
Theorem 2.2. Let $\left(\mathcal{B},\|\cdot\|_{\mathcal{B}}\right)$ be a Banach algebra which contains the separable Banach algebra $\left(\mathcal{A},\|\cdot\|_{\mathcal{A}}\right)$ as a two-sided ideal such that $\|\cdot\|_{\mathcal{B}} \leq\|\cdot\|_{\mathcal{A}}$ and let $\phi \in \Delta(\mathcal{A})$. Then the following statements are equivalent.

(a) There is $b \in S_{\phi}^{\mathcal{B}}$ such that $H_{b, \phi}=\mathbb{C} \phi$.

(b) $\mathcal{A}$ has a $\phi$-mean in $\overline{S_{\phi}^{\mathcal{A}} w^{*}}$.

(c) There is $b \in S_{\phi}^{\mathcal{A}}$ such that $H_{b, \phi}=\mathbb{C} \phi$.

Proof. (a) $\Rightarrow$ (b). Suppose that $H_{b, \phi}=\mathbb{C} \phi$ for some $b \in S_{\phi}^{\mathcal{B}}$. Then it follows from $I_{b, \phi} \subseteq I_{\phi}$ and $I_{\phi}^{\perp}=\mathbb{C} \phi$ that $I_{b, \phi}=I_{\phi}$. Now, for each $n \in \mathbb{N}$ consider the element

$$
b_{n}=\frac{1}{n} \sum_{j=1}^{n} b^{j}
$$

in $S_{\phi}^{\mathcal{B}}$. Thus for each $a \in \mathcal{A}$ we have

$$
\lim _{n \rightarrow \infty}\left\|(a-a b) b_{n}\right\|_{\mathcal{A}} \leq \lim _{n \rightarrow \infty} \frac{2}{n}\|a\|_{\mathcal{A}}=0 .
$$

Since $I_{\phi}=I_{b, \phi}$, it follows that

$$
\lim _{n \rightarrow \infty}\left\|a b_{n}\right\|_{\mathcal{A}}=0
$$

for all $a \in I_{\phi}$. Choose $b_{0} \in S_{\phi}^{\mathcal{A}}$. Then $a b_{0}-\phi(a) b_{0} \in I_{\phi}$ for all $a \in \mathcal{A}$. For each $n \in \mathbb{N}$ define $a_{n}:=b_{0} b_{n}$. Thus, $\left(a_{n}\right) \subseteq S_{\phi}^{\mathcal{A}}$ and for each $a \in \mathcal{A}$,

$$
\lim _{n \rightarrow \infty}\left\|a a_{n}-\phi(a) a_{n}\right\|_{\mathcal{A}}=\lim _{n \rightarrow \infty}\left\|\left(a b_{0}-\phi(a) b_{0}\right) b_{n}\right\|_{\mathcal{A}}=0 .
$$

It is clear that any weak* cluster point of $\left(a_{n}\right)$ is a $\phi$-mean in $\overline{S_{\phi}^{\mathcal{A}}} w^{*}$.

(b) $\Rightarrow$ (c). Suppose that (b) holds. Then there is an element $b \in S_{\phi}^{\mathcal{A}}$ such that $\left\|a b^{n}-\phi(a) b^{n}\right\| \rightarrow 0$ for all $a \in \mathcal{A}$ by Lemma 2.1. It is easy to see that $b^{n} \cdot f=f$ for all $f \in H_{b}$ and $n \in \mathbb{N}$. Thus,

$$
\begin{aligned}
\left\langle\left(f-f\left(b^{n}\right) \phi\right), a\right\rangle & =\left\langle\left(b^{n} \cdot f-f\left(b^{n}\right) \phi\right), a\right\rangle \\
& =\left\langle f, a b^{n}-\phi(a) b^{n}\right\rangle \rightarrow 0
\end{aligned}
$$

for all $a \in \mathcal{A}$. This shows that $f\left(b^{n}\right) \phi \rightarrow f$ in the weak ${ }^{*}$ topology of $\mathcal{A}^{*}$ and consequently $f \in \mathbb{C} \phi$, as required.

The implication (c) $\Rightarrow$ (a) is trivial.

Remark 2.3. Recall that a Lau algebra $\mathcal{A}$ is a Banach algebra which is the predual of von Neumann algebra $\mathcal{M}$ such that the identity element $\epsilon$ of $\mathcal{M}$ is a multiplicative linear functional on $\mathcal{A}$. In this case, the $\epsilon$-means of norm one are nothing but the topological left invariant means on $\mathcal{A}^{*}$; see [14] for details. $\mathcal{A}$ is called left amenable if there is a topological left invariant mean on $\mathcal{A}^{*}$. Examples of Lau algebras include the group algebra $L^{1}(G)$ of a locally compact group or hypergroup $G$, the Fourier algebra and the Fourier-Stieltjes algebra of a locally compact group. Other examples are the measure algebra $M(S)$ of a locally compact semi-topological semigroup or hypergroup $S$ and the predual of a Hopf-von Neumann algebra. For a more recent example of Lau algebras, consider the Fourier-Stieltjes algebra of a topological group as defined in [16]. For a Lau 
algebra $\mathcal{A}$, the $\epsilon$-maximal elements are precisely the positive linear functionals of norm one in $\mathcal{A}$ and hence span $\mathcal{A}$. In view of [15, Lemma 2.1], the set of states in the predual of a von Neumann algebra is weak* dense in the set of states in its dual space. In particular,

$$
\overline{S_{\epsilon}^{\mathcal{A}}} w^{*}=\left\{F \in \mathcal{A}^{* *}:\|F\|=F(\epsilon)=1\right\} .
$$

Thus, by Lemma 2.1 and Theorem 2.2 for a separable Lau algebra $\mathcal{A}$ the following statements are equivalent.

(a) $\mathcal{A}$ is left amenable.

(b) There is a state $b$ in $\mathcal{A}$ such that $\left\|a b^{n}-\epsilon(a) b^{n}\right\| \rightarrow 0$ for all $a \in \mathcal{A}$.

(c) There is a state $b$ in $\mathcal{A}$ such that $H_{b, \epsilon}=\mathbb{C} \epsilon$.

For any $T \in P M_{p}(G)$ we denote by supp $T$ the support of $T$ which is defined as follows: $x \in \operatorname{supp} T$ if $\phi_{x}$ is the weak* limit of operators $T \cdot v$, where $v \in A_{p}(G)$ or equivalently, $x \in \operatorname{supp} T$ if and only if there is a net $\left(u_{\alpha}\right)$ in $A_{p}(G)$ such that $u_{\alpha} \cdot T \rightarrow \phi_{x}$ in the weak ${ }^{*}$ topology of $P M_{p}(G)$; see for details [13, p.267] and [7, Proposition 10].

If $G$ is first countable and $1<p<\infty$, then by a same argument for the case $p=2$; see [5, Corollary 6.9], we can show that $A_{p}(G)$ is norm separable. Following [20] for each $x \in G$, we call $\sigma \in S_{x}^{M}$ adapted if $\{y \in G: \sigma(y)=1\}=\{x\}$.

Theorem 2.4. Let $G$ be a locally compact group and let $x \in G$. Then the following statements are equivalent.

(a) There is an adapted $\sigma \in S_{x}^{M}$

(b) $G$ is first countable.

(c) There is $\sigma \in S_{x}^{M}$ such that $\left\|v \sigma^{n}\right\|_{A_{p}} \rightarrow 0$ for all $v \in I_{x}$.

(d) There is $\sigma \in S_{x}^{M}$ such that $H_{\sigma, x}=\mathbb{C} \phi_{x}$.

(e) There is $\sigma \in S_{x}^{M}$ such that $I_{x}=I_{\sigma, x}$.

(f) There is an adapted $\sigma \in S_{x}^{A}$.

Proof. $(\mathrm{a}) \Rightarrow(\mathrm{b})$. Suppose that $\sigma \in S_{x}^{M}$ is adapted and let $U$ be a compact neighborhood of $e$. For each $n \in \mathbb{N}$ define

$$
U_{n}=\left\{x \in U:|\sigma(x)-1|<\frac{1}{n}\right\} .
$$

Continuity of $\sigma$ implies that $\left\{U_{n}: n \in \mathbb{N}\right\}$ consists of neighborhoods of $e$. Let $V$ be a compact neighborhood of $e$, without loss of generality we can assume that $V$ is open and $V \subseteq U$. Let

$$
d=\inf \{|\sigma(x)-1|: x \in U \backslash V\} .
$$

Since $U \backslash V$ is compact and $\sigma$ is adapted and continuous, it follows that $d>0$. We can find $m \in \mathbb{N}$ such that $\frac{1}{m} \leq d$. Thus $U_{n} \subseteq V$ for all $n \geq m$. This shows that $\left\{U_{n}: n \in \mathbb{N}\right\}$ is a base of neighborhoods of $e$ and so $G$ is first countable.

Implications $(\mathrm{b}) \Rightarrow(\mathrm{c})$ and $(\mathrm{b}) \Rightarrow(\mathrm{d})$ follow from Lemma 2.1 and Theorem 2.2.

$(\mathrm{d}) \Leftrightarrow(\mathrm{e})$. This follows from $I_{\sigma, x} \subseteq I_{x}$ and $I_{x}^{\perp}=\mathbb{C} \phi_{x}$.

$(\mathrm{e}) \Rightarrow(\mathrm{f})$. Let $T \in H_{\sigma, x}$ and choose $y \in \operatorname{supp} T$. Then there is a net $\left(u_{\alpha}\right) \subseteq A_{p}(G)$ such that $u_{\alpha} \cdot T \stackrel{w^{*}}{\rightarrow} \phi_{y}$. Moreover, $\sigma \cdot\left(u_{\alpha} \cdot T\right)=u_{\alpha} \cdot T$ for all $\alpha$. Now, given $u_{0} \in S_{y}$. 
Then we have

$$
\begin{aligned}
\lim _{\alpha}\left\langle u_{\alpha} \cdot T, u_{0}\right\rangle & =\lim _{\alpha}\left\langle\sigma \cdot\left(u_{\alpha} \cdot T\right), u_{0}\right\rangle \\
& =\left\langle\sigma \cdot \phi_{y}, u_{0}\right\rangle \\
& =\sigma(y) .
\end{aligned}
$$

On the other hand,

$$
\begin{aligned}
\lim _{\alpha}\left\langle u_{\alpha} \cdot T, u_{0}\right\rangle & =\phi_{y}\left(u_{0}\right) \\
& =u_{0}(y) \\
& =1 .
\end{aligned}
$$

Therefore, $y=x$ by assumption and so $\operatorname{supp} T=\{x\}$. Thus $T \in \mathbb{C} \phi_{x}$.

Finally, (f) $\Rightarrow($ a) is trivial.

A group $G$ is called amenable if there exists a continuous linear functional $m \in L^{\infty}(G)^{*}$ such that $m\left(L_{a} f\right)=m(f)$ for all $f \in L^{\infty}(G)$ and $a \in G$. It is well known that $A_{p}(G)$ has a bounded approximate identity if and only if $G$ is amenable. Now, we have the following lemma whose proof is omitted, since it can be proved similarly to [3, Lemma 3.2.2].

Lemma 2.5. Let $G$ be an amenable locally compact group and let $x \in G$. Then $I_{\sigma, x}$ has a bounded approximate identity for all $\sigma \in S_{x}^{M}$.

We recall that for each $x \in G$ the ideal $I_{x}$ has a bounded approximate identity if and only if $G$ is amenable; see for example either [18, Proposition 3.9] or [11, Corollary 2.3]. Thus, we have the following result by Lemma 2.5 and Theorem 2.4 .

Proposition 2.6. Let $G$ be a first countable locally compact group. Then $G$ is amenable if and only if $I_{\sigma, x}$ has a bounded approximate identity for all $\sigma \in S_{x}^{M}$.

Acknowledgement. The author would like to sincerely thank the referee of the paper for the valuable comments and constructive suggestions. He acknowledges that this research was partially carried out at the IPM-Isfahan Branch. This research was in part supported by a grant from IPM (No. 92470046)

\section{REFERENCES}

1. F. Abtahi, Generalized biprojectivity and biflatness of abstract Segal algebras, Banach J. Math. Anal. 8 (2014), 107-117.

2. M. Alaghmandan, R. Nasr-Isfahani, and M. Nemati, Character amenability and contractibility of abstract Segal algebras, Bull. Austral. Math. Soc. 82 (2010), 274-281.

3. C.H. Chu and A.T. Lau, Harmonic functions on groups and Fourier algebras, Lecture Notes in Math., vol. 1782, Springer-Verlag, Berlin, 2002.

4. P. Eymard, L, algébre de Fourier d'un groupe localement compact, Bull. Soc. Math. France 92 (1964), 181-236.

5. C.C. Graham, A.T. Lau and M. Leinert, Separable translation invariant subspaces of $M(G)$ and other dual spaces on locally compact groups, Colloq. Math. 55 (1988), 131-145.

6. C. Herz, The theory of p-spaces with application to convolution operators, Trans. Amer. Math. Soc. 154 (1971), 69-82.

7. C.S. Herz, Harmonic synthesis on subgroups, Ann. Inst. Fourier 23 (1973), 91-123. 
8. Z. Hu, Spectrum of commutative Banach algebras and isomorphism of $C^{*}$-algebras related to locally compact groups, Studia Math. 129 (1998), 207-223.

9. Z. Hu, M.S. Monfared and T. Traynor, On character amenable Banach algebras, Studia Math. 193 (2009) 53-78.

10. M. Kalantar, M. Neufang and Z.-J. Ruan, Poisson boundaries over locally compact quantum groups, Internat. J. Math. 24 (2013), no. 3, 1350023, 21 pp.

11. E. Kaniuth, A.T. Lau and J. Pym, On $\phi$-amenability of Banach algebras, Math. Proc. Cambridge Philos. Soc. 144 (2008), 85-96.

12. E. Kaniuth, A.T. Lau and J. Pym, On character amenability of Banach algebras, J. Math. Anal. Appl. 344 (2008), 942-955.

13. E. Kaniuth, A.T. Lau and A. Ulger, Multipliers of commutative Banach algebras, power boundedness and Fourier-Stieltjes algebras, J. London Math. Soc. 81 (2010), 255-275.

14. A.T. Lau, Analysis on a class of Banach algebras with application to harmonic analysis on locally compact groups and semigroups, Fund. Math. 118 (1983), 161-175.

15. A.T. Lau, Uniformly continuous functionals on Banach algebras, Colloq. Math. 51 (1987), 195-205.

16. A.T. Lau and J. Ludwig, Fourier-Stieltjes algebra of a topological group, Adv. Math. 229 (2012), 2000-2023

17. F. Lust-Piquard, Means on $C V_{p}(G)$-subspaces of $C V_{p}(G)$ with RNP and Schur property, Ann. Inst. Fourier, 39 (1989), 969-1006.

18. M.S. Monfared, Extensions and isomorphisms for the generalized Fourier algebras of a locally compact group, J. Funct. Anal. 198 (2003), 413-444.

19. M.S. Monfared, Character amenability of Banach algebras, Math. Proc. Cambridge Philos. Soc. 144 (2008), 697-706.

20. M. Neufang and V. Runde, Harmonic operators: the dual perspective, Math. Z. 255 (2007), 669-690.

Department of Mathematical Sciences, Isfahan Uinversity of Technology, IsFAHAN 84156-83111, IRAN;

School of Mathematics, Institute for Research in Fundamental Sciences (IPM), P.O. Box: 19395-5746, TEHRAN, Iran.

E-mail address: m.nemati@cc.iut.ac.ir 